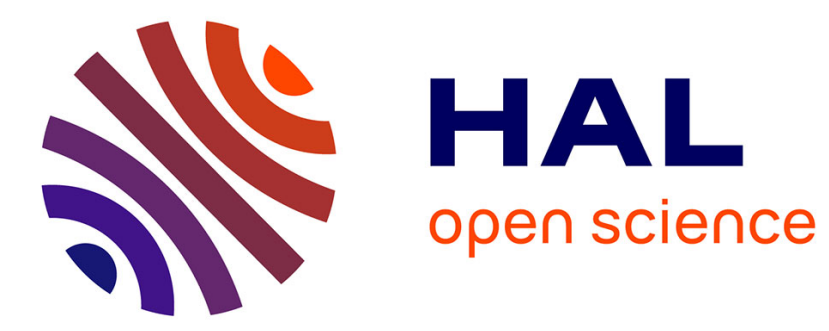

\title{
Analysis of a discontinuous Galerkin method for heterogeneous diffusion problems with low-regularity solutions
}

\author{
Daniele Antonio Di Pietro, Alexandre Ern
}

\section{To cite this version:}

Daniele Antonio Di Pietro, Alexandre Ern. Analysis of a discontinuous Galerkin method for heterogeneous diffusion problems with low-regularity solutions. Numerical Methods for Partial Differential Equations, 2011, 17 p. 10.1002/num.20675 . hal-00514387

\author{
HAL Id: hal-00514387 \\ https://hal.science/hal-00514387
}

Submitted on 2 Sep 2010

HAL is a multi-disciplinary open access archive for the deposit and dissemination of scientific research documents, whether they are published or not. The documents may come from teaching and research institutions in France or abroad, or from public or private research centers.
L'archive ouverte pluridisciplinaire HAL, est destinée au dépôt et à la diffusion de documents scientifiques de niveau recherche, publiés ou non, émanant des établissements d'enseignement et de recherche français ou étrangers, des laboratoires publics ou privés. 


\title{
ANALYSIS OF A DISCONTINUOUS GALERKIN METHOD FOR HETEROGENEOUS DIFFUSION PROBLEMS WITH LOW-REGULARITY SOLUTIONS
}

\author{
DANIELE A. DI PIETRO ${ }^{1}$ AND ALEXANDRE ERN ${ }^{2}$
}

\begin{abstract}
We study the convergence of the Symmetric Weighted Interior Penalty discontinuous Galerkin method for heterogeneous diffusion problems with low-regularity solutions only belonging to $W^{2, p}$ with $p \in(1,2]$. In $2 \mathrm{~d}$ we infer an optimal algebraic convergence rate. In $3 \mathrm{~d}$ we achieve the same result for $p>6 / 5$, and for $p \in(1,6 / 5]$ we prove convergence without algebraic rate.
\end{abstract}

\section{INTRODUCTION}

In this work we analyze the convergence of a discontinuous Galerkin (dG) approximation to low-regularity solutions of the model problem

$$
\begin{aligned}
-\nabla \cdot(\kappa \nabla u)=f & \text { in } \Omega, \\
u=0 & \text { on } \partial \Omega,
\end{aligned}
$$

where, for $d \in\{2,3\}, \Omega$ denotes a bounded connected polyhedral domain with boundary $\partial \Omega, f \in L^{2}(\Omega)$ is the forcing term, and $\kappa \in L^{\infty}(\Omega)$ is the diffusion coefficient such that $\underline{\lambda} \leq \kappa \leq \bar{\lambda}$ a.e. in $\Omega$ for positive real numbers $\underline{\lambda}$ and $\bar{\lambda}$. Owing to the Lax-Milgram Lemma, this problem is well-posed in the energy space $V:=H_{0}^{1}(\Omega)$.

In practice, the diffusion coefficient has more regularity than just belonging to $L^{\infty}(\Omega)$. In what follows, we assume that there is a partition $P_{\Omega}:=\left\{\Omega_{i}\right\}_{1 \leq i \leq N_{\Omega}}$ of $\Omega$ such that

(i) each $\Omega_{i}, 1 \leq i \leq N_{\Omega}$, is an open polyhedron;

(ii) the restriction of $\kappa$ to each $\Omega_{i}, 1 \leq i \leq N_{\Omega}$, is constant.

The regularity of the exact solution for interface problems matching the above assumption has been studied by Nicaise and Sändig [13], where it is proven that

$$
\text { There exists } p \in(1,2] \text { s.t. } u \in V_{\dagger}:=W^{2, p}\left(P_{\Omega}\right) \text {, }
$$

where $W^{2, p}\left(P_{\Omega}\right)$ denotes the broken Sobolev space spanned by those functions $v$ such that $\left.v\right|_{\Omega_{i}} \in W^{2, p}\left(\Omega_{i}\right)$ for all $1 \leq i \leq N_{\Omega}$. However, up to date, the convergence analysis of dG methods for the interface problem (1) has generally hinged on a more stringent regularity assumption on the exact solution, namely $u \in H^{3 / 2+\epsilon}\left(P_{\Omega}\right)$ with $\epsilon>0$. The goal of this paper is to fill the gap by using only the regularity (2). We fully achieve this goal in $2 \mathrm{~d}$, whereby we derive energy norm error estimates with optimal algebraic convergence rates. A similar result has been established recently by Wihler and Rivière [18] in the simpler case of the Laplace equation in 2d. As

Date: Draft version: September 2, 2010. 
in [18] our analysis hinges on discrete stability, strong consistency and boundedness of the discrete bilinear form, but handling the heterogeneity of the diffusion coefficient requires special care to achieve robustness. The boundedness property is also formulated in a somewhat different way. In $3 \mathrm{~d}$ the situation is more delicate. For $p \in(6 / 5,2]$ we also derive optimal algebraic convergence rates for the energy norm error. In this case, owing to the Sobolev embedding, the exact solution is indeed in $H^{1+\alpha}\left(P_{\Omega}\right)$ with $\alpha>0$. For brevity, we treat the $2 \mathrm{~d}$ case with $p \in(1,2]$ and the $3 \mathrm{~d}$ case with $p \in(6 / 5,2]$ simultaneously; the analysis readily extends to $p \in(2 d / d+2,2]$ in any space dimension. Finally, in $3 \mathrm{~d}$ with $p \in(1,6 / 5]$, we present for the sake of completeness a convergence proof without algebraic rates. The analysis, valid in any space dimension, follows the compactness argument introduced in [5]. Herein, we consider shape-regular meshes. An alternative approach based on geometrically refined meshes has been investigated, e.g., by Wihler, Frauenfelder, and Schwab [17].

The focus is here on the Symmetric Weighted Interior Penalty (SWIP) dG method to approximate the model problem (1) (accounting for variations in symmetry is straightforward). The SWIP method has been introduced in the more general context of diffusion-advection-reaction problems by Di Pietro, Ern, and Guermond [6] and Ern, Stephansen, and Zunino [9]. For the model problem (1), the differences with respect to the classical Symmetric Interior Penalty (SIP) method of Arnold [2] lay in the use of diffusion-dependent, weighted average trace operators and of a penalty parameter proportional to the harmonic average of the diffusion at interfaces. This allows one to infer energy norm error estimates with multiplicative constant independent of diffusion heterogeneity, which makes the SWIP method particularly suited to diffusion-advection problems with sharp internal layers. The possibility of using non-arithmetic averages in dG methods has been pointed out and used in various contexts, e.g., by Stenberg [14] and by Heinrich and co-workers $[11,10,12]$. The idea of connecting the actual value of the weights to the diffusion coefficient was originally proposed by Burman and Zunino [4] in the context of mortaring techniques for a singularly perturbed diffusion-advection equation.

The material is organized as follows. In $\S 2$ we present the discrete setting. In $\$ 3$ we derive algebraic convergence rates for exact solutions in $W^{2, p}\left(P_{\Omega}\right)$ with $p \in(2 d / d+2,2]$. Finally, the convergence for the remaining cases is covered in $\S 4$. Numerical results have already been presented in $[8,15]$ for the well-known $2 \mathrm{~d}$ fourcorner problem, whereby the convergence rates derived herein have been observed numerically.

\section{The Discrete SETTING}

2.1. Meshes and faces. Let $\left(\mathcal{T}_{h}\right)_{h \in \mathcal{H}}$ be a sequence of refined simplicial meshes covering $\Omega$ exactly, where $\mathcal{H}$ denotes a countable set having zero as unique accumulation point. Meshes can possess hanging nodes. Quite importantly, meshes are assumed to be compatible with the partition $P_{\Omega}$, that is, such that for all $h \in \mathcal{H}$ and all $T \in \mathcal{T}_{h}$, there exists a unique $\Omega_{i}$ of the partition $P_{\Omega}$ such that $T \subset \Omega_{i}$. Since the diffusion coefficient is piecewise constant on the partition $P_{\Omega}$, it is also piecewise constant on each compatible mesh.

For a mesh element $T \in \mathcal{T}_{h}, h_{T}$ denotes its diameter and $\mathrm{n}_{T}$ its unit outward normal defined a.e. on $\partial T$. The mesh-size is $h:=\max _{T \in \mathcal{T}_{h}} h_{T}$. The following 


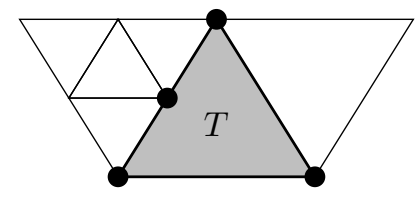

Figure 1. The set $\mathcal{F}_{T}$ for the element $T$ (shaded) contains in this case the four mesh faces with vertices in bold line

definitions apply for every $h \in \mathcal{H}$. For every integer $k \geq 0$, we introduce the space

$$
\mathbb{P}_{d}^{k}\left(\mathcal{T}_{h}\right):=\left\{v_{h} \in L^{2}(\Omega)\left|\forall T \in \mathcal{T}_{h}, v_{h}\right|_{T} \in \mathbb{P}_{d}^{k}(T)\right\},
$$

where $\mathbb{P}_{d}^{k}(T)$ in spanned by the restriction to $T$ of polynomial functions in $d$ variables of total degree $\leq k$. We say that a (closed) subset $F$ of $\bar{\Omega}$ is a mesh face if $F$ has positive $(d-1)$-dimensional measure and if one of the two following mutually exclusive conditions is satisfied:

(i) There are distinct mesh elements $T_{1}, T_{2} \in \mathcal{T}_{h}$ such that $F=\partial T_{1} \cap \partial T_{2}$; in such case, $F$ is called an interface and we set $\mathrm{n}_{F}:=\mathrm{n}_{T_{1}}$, the unit normal vector to $F$ pointing from $T_{1}$ to $T_{2}$ (the orientation of $\mathrm{n}_{F}$ is arbitrary depending on the choice of $T_{1}$ and $T_{2}$, but kept fixed in what follows);

(ii) There is $T \in \mathcal{T}_{h}$ such that $F=\partial T \cap \partial \Omega$; in such case, $F$ is called a boundary face and we set $\mathrm{n}_{F}:=\mathrm{n}$, the outward unit normal to $\partial \Omega$.

Interfaces are collected in the set $\mathcal{F}_{h}^{\mathrm{i}}$, boundary faces in $\mathcal{F}_{h}^{\mathrm{b}}$, and mesh faces in $\mathcal{F}_{h}:=\mathcal{F}_{h}^{\mathrm{i}} \cup \mathcal{F}_{h}^{\mathrm{b}}$. Moreover, for every mesh element $T \in \mathcal{T}_{h}$, the set

$$
\mathcal{F}_{T}:=\left\{F \in \mathcal{F}_{h} \mid F \subset \partial T\right\}
$$

contains the mesh faces composing the boundary of $T$. As nonmatching meshes are allowed, the cardinal number of $\mathcal{F}_{T}$ can be larger than $(d+1)$; see Figure 1. In what follows, we assume that $\left(\mathcal{T}_{h}\right)_{h \in \mathcal{H}}$ is an admissible mesh sequence, that is, $\mathcal{T}_{h}$ is shape-regular in the usual sense and contact-regular meaning that there exists $C$ independent of the mesh-size $h$ such that, for all $T \in \mathcal{T}_{h}$ and all $F \in \mathcal{F}_{T}, h_{T} \leq C h_{F}$, the diameter of $F$. Letting

$$
N_{\partial}:=\max _{h \in \mathcal{H}, T \in \mathcal{T}_{h}} \operatorname{card}\left(\mathcal{F}_{T}\right),
$$

contact regularity implies that $N_{\partial}$ is bounded.

\subsection{Jumps and weighted averages.}

Definition 2.1 (Jumps). Let $v$ be a scalar-valued function defined on $\Omega$ and assume that $v$ is smooth enough to admit on all $F \in \mathcal{F}_{h}$ a (possibly two-valued) trace. Then, if $F \in \mathcal{F}_{h}^{\mathrm{i}}$ with $F=\partial T_{1} \cap \partial T_{2}$, the jump of $v$ at $F$ is defined for a.e. $x \in F$ as

$$
\llbracket v \rrbracket_{F}(x):=\left.v\right|_{T_{1}}(x)-\left.v\right|_{T_{2}}(x),
$$

while if $F \in \mathcal{F}_{h}^{\mathrm{b}}$ with $F=\partial T \cap \partial \Omega$, we set $\llbracket v \rrbracket_{F}(x):=\left.v\right|_{T}(x)$.

Definition 2.2 (Weighted averages). Let $v$ be a scalar-valued function defined on $\Omega$ and assume that $v$ is smooth enough to admit on all $F \in \mathcal{F}_{h}$ a (possibly two-valued) trace. To any interface $F \in \mathcal{F}_{h}^{\mathrm{i}}$ with $F=\partial T_{1} \cap \partial T_{2}$, we assign two non-negative real numbers $\omega_{T_{1}, F}$ and $\omega_{T_{2}, F}$ such that

$$
\omega_{T_{1}, F}+\omega_{T_{2}, F}=1
$$


Then, the weighted average of $v$ at $F \in \mathcal{F}_{h}^{\mathrm{i}}$ is defined for a.e. $x \in F$ as

$$
\{v\}_{\omega, F}(x):=\left.\omega_{T_{1}, F} v\right|_{T_{1}}(x)+\left.\omega_{T_{2}, F} v\right|_{T_{2}}(x) .
$$

while on boundary faces $F \in \mathcal{F}_{h}^{\mathrm{b}}$ with $F=\partial T \cap \partial \Omega$, we set $\{v\}_{\omega, F}(x):=\left.v\right|_{T}(x)$.

Clearly, the usual (arithmetic) average at interfaces corresponds to the particular choice $\omega_{T_{1}, F}=\omega_{T_{1}, F}=\frac{1}{2}$. Henceforth, we consider a specific diffusion-dependent choice for the weights, namely for all $F \in \mathcal{F}_{h}^{\mathrm{i}}, F=\partial T_{1} \cap \partial T_{2}$,

$$
\omega_{T_{1}, F}:=\frac{\kappa_{2}}{\kappa_{1}+\kappa_{2}}, \quad \omega_{T_{2}, F}:=\frac{\kappa_{1}}{\kappa_{1}+\kappa_{2}},
$$

where $\kappa_{i}=\left.\kappa\right|_{T_{i}}, i \in\{1,2\}$. In particular, the case of homogeneous diffusion yields the usual (arithmetic) averages. When $v$ is vector-valued, the above average and jump operators act componentwise. Whenever no confusion can arise, both the subscript $F$ and the variable $x$ are omitted.

2.3. The discrete problem. We aim at approximating the exact solution $u$ of (1) by a dG method using the discrete space

$$
V_{h}:=\mathbb{P}_{d}^{k}\left(\mathcal{T}_{h}\right), \quad k \geq 1 .
$$

Define for all $\left(v_{h}, w_{h}\right) \in V_{h} \times V_{h}$,

$$
\begin{aligned}
a_{h}\left(v_{h}, w_{h}\right):= & \int_{\Omega} \kappa \nabla_{h} v_{h} \cdot \nabla_{h} w_{h}+\sum_{F \in \mathcal{F}_{h}} \eta \frac{\gamma_{\kappa, F}}{h_{F}} \int_{F} \llbracket v_{h} \rrbracket \llbracket w_{h} \rrbracket \\
& -\sum_{F \in \mathcal{F}_{h}} \int_{F}\left\{\kappa \nabla_{h} v_{h}\right\}_{\omega} \cdot \mathrm{n}_{F} \llbracket w_{h} \rrbracket-\sum_{F \in \mathcal{F}_{h}} \int_{F} \llbracket v_{h} \rrbracket\left\{\kappa \nabla_{h} w_{h}\right\}_{\omega} \cdot \mathrm{n}_{F},
\end{aligned}
$$

where $\nabla_{h}$ denotes the usual broken gradient operator on $\mathcal{T}_{h}, \eta>0$ is a userdependent penalty parameter (to be chosen large enough to ensure discrete stability, see Lemma 3.4), while the diffusion-dependent penalty parameter $\gamma_{\kappa, F}$ is such that for all $F \in \mathcal{F}_{h}^{\mathrm{i}}$ with $F=\partial T_{1} \cap \partial T_{2}$,

$$
\gamma_{\kappa, F}:=\frac{2 \kappa_{1} \kappa_{2}}{\kappa_{1}+\kappa_{2}},
$$

where, as above, $\kappa_{i}=\left.\kappa\right|_{T_{i}}, i \in\{1,2\}$, while for all $F \in \mathcal{F}_{h}^{\mathrm{b}}, F=\partial T \cap \partial \Omega$,

$$
\gamma_{\kappa, F}:=\left.\kappa\right|_{T} .
$$

We notice that the above choice for the penalty parameter $\gamma_{\kappa, F}$ on interfaces corresponds to the harmonic mean of the two diffusion coefficients on either side of the interface. In what follows, the terms in the second line of (4) are respectively referred to as consistency and symmetry terms, as they serve the enforcement of the corresponding property at the discrete level. The bilinear form $a_{h}$ defined by (4) is termed the Symmetric Weighted Interior Penalty (SWIP) bilinear form [6, 9]. Whenever $\kappa$ is constant in $\Omega$, the usual (arithmetic) averages are recovered in the consistency and symmetry terms. Finally, the discrete problem is

$$
\text { Find } u_{h} \in V_{h} \text { s.t. } a_{h}\left(u_{h}, v_{h}\right)=\int_{\Omega} f v_{h} \text { for all } v_{h} \in V_{h} \text {. }
$$


2.4. Extension of the discrete bilinear form. To assert consistency for the discrete problem (5) in the usual strong form, we need to plug the exact solution $u$ into the first argument of the bilinear form $a_{h}$. This requires in turn to give a meaning to the normal gradient of $u$ independently on each mesh face. The fact that $-\Delta u=f \in L^{2}(T)$ for all $T \in \mathcal{T}_{h}$ is insufficient, as it only yields $\nabla u \cdot \mathrm{n}_{T} \in$ $H^{-1 / 2}(\partial T)$. The regularity (2) is thus crucial, since owing to mesh compatibility, it implies for all $v \in V_{\dagger}$, all $T \in \mathcal{T}_{h}$, and all $F \in \mathcal{F}_{T}$,

$$
\nabla v \cdot \mathrm{n}_{T} \in L^{p}(F) .
$$

As a result, the discrete bilinear form $a_{h}$ can be extended to $V_{\dagger h} \times V_{h}$ with

$$
V_{\dagger h}:=V_{\dagger}+V_{h},
$$

and $V_{\dagger}$ defined by (2).

\section{Convergence analysis in 2D AND IN 3D FOR $p \in(6 / 5,2]$}

In this section we prove optimal convergence rates for the method (5) in $2 \mathrm{~d}$ and in $3 \mathrm{~d}$ for $p>6 / 5$, that is, $p>2 d / d+2$. Owing to the Sobolev embedding theorem, the regularity (2) yields

$$
u \in H^{1+\alpha}\left(P_{\Omega}\right) \text { with } \alpha:=1+d\left(\frac{1}{2}-\frac{1}{p}\right)>0 .
$$

The error analysis in this section proceeds by establishing consistency, discrete stability, and boundedness for the SWIP bilinear form $a_{h}$. The error is measured in the following energy norm: For all $v \in V_{\dagger h}$,

$$
\|v\|_{\kappa}:=\left(\left\|\kappa^{1 / 2} \nabla_{h} v\right\|_{\left[L^{2}(\Omega)\right]^{d}}^{2}+|v|_{\mathrm{J}, \kappa}^{2}\right)^{1 / 2},
$$

with jump seminorm

$$
|v|_{\mathrm{J}, \kappa}:=\left(\sum_{F \in \mathcal{F}_{h}}|v|_{\mathrm{J}, \kappa, F}^{2}\right)^{1 / 2}, \quad|v|_{\mathrm{J}, \kappa, F}:=\left(\frac{\gamma_{\kappa, F}}{h_{F}}\right)^{1 / 2}\|\llbracket v \rrbracket\|_{L^{2}(F)} .
$$

3.1. Technical results. This section collects some useful technical results. We recall the following inverse and trace inequalities (see, e.g., [3, 7]): For all $y_{h} \in V_{h}$ and all $F \in \mathcal{F}_{h}$,

$$
\left\|y_{h}\right\|_{L^{q}(F)} \leq C_{q} h_{F}^{(d-1)\left(\frac{1}{q}-\frac{1}{2}\right)}\left\|y_{h}\right\|_{L^{2}(F)},
$$

and the following trace inequality: For all $y_{h} \in V_{h}$, all $T \in \mathcal{T}_{h}$, and all $F \in \mathcal{F}_{T}$,

$$
h_{F}^{1 / 2}\left\|y_{h}\right\|_{L^{2}(F)} \leq C_{\mathrm{tr}}\left\|y_{h}\right\|_{L^{2}(T)} .
$$

The quantity $C_{\mathrm{tr}}$ only depends on $d, k$, and mesh regularity, while there holds $C_{q} \leq \max \left(1, C_{\infty}\right)[16]$ where $C_{\infty}$ only depends on $d, k$, and mesh regularity. For a real number $r \in(1,+\infty)$, we set

$$
\beta_{r}:=\frac{1}{2}+(d-1)\left(\frac{1}{2}-\frac{1}{r}\right),
$$

and observe that for $r=2, \beta_{2}=\frac{1}{2}$. We consider the following seminorm

$$
|v|_{\dagger, \kappa, r}:=\left(\sum_{T \in \mathcal{T}_{h}} \sum_{F \in \mathcal{F}_{T}} h_{F}^{r \beta_{r}}\left\|\left.\kappa^{1 / 2} \nabla v\right|_{T} \cdot \mathrm{n}_{F}\right\|_{L^{r}(F)}^{r}\right)^{1 / r} .
$$


In particular, for $r=2$,

$$
|v|_{\dagger, \kappa, 2}=\left(\sum_{T \in \mathcal{T}_{h}} \sum_{F \in \mathcal{F}_{T}} h_{F}\left\|\left.\kappa^{1 / 2} \nabla v\right|_{T} \cdot \mathrm{n}_{F}\right\|_{L^{2}(F)}^{2}\right)^{1 / 2} .
$$

The main result of this section is a bound on the consistency and symmetry terms in the SWIP bilinear form $a_{h}$. In what follows, we set $q:=\frac{p}{p-1}$ so that $\frac{1}{p}+\frac{1}{q}=1$ and $q \in[2,+\infty)$.

Lemma 3.1 (Bound on consistency and symmetry terms). There holds:

(i) For all $\left(v_{h}, w\right) \in V_{h} \times V_{\dagger h}$,

$$
\left|\sum_{F \in \mathcal{F}_{h}} \int_{F}\left\{\kappa \nabla_{h} v_{h}\right\}_{\omega} \cdot \mathrm{n}_{F} \llbracket w \rrbracket\right| \leq\left|v_{h}\right|_{\dagger, \kappa, 2}|w|_{\mathrm{J}, \kappa} .
$$

(ii) For all $\left(v, w_{h}\right) \in V_{\dagger h} \times V_{h}$,

$$
\left|\sum_{F \in \mathcal{F}_{h}} \int_{F}\left\{\kappa \nabla_{h} v\right\}_{\omega} \cdot \mathrm{n}_{F} \llbracket w_{h} \rrbracket\right| \leq 2^{\frac{1}{2}-\frac{1}{q}} C_{q}|v|_{\dagger, \kappa, p}\left|w_{h}\right|_{\mathrm{J}, \kappa} .
$$

Proof. (i) Proof of (12). Let $\left(v_{h}, w\right) \in V_{h} \times V_{\dagger h}$. For all $F \in \mathcal{F}_{h}^{\mathrm{i}}$ with $F=\partial T_{1} \cap \partial T_{2}$, set $\omega_{i}=\omega_{T_{i}, F}, \kappa_{i}=\left.\kappa\right|_{T_{i}}$, and $a_{i}=\left.\kappa_{i}^{1 / 2}\left(\nabla_{h} v_{h}\right)\right|_{T_{i}} \cdot \mathrm{n}_{F}, i \in\{1,2\}$. The CauchySchwarz inequality yields

$$
\begin{aligned}
\int_{F}\left\{\kappa \nabla_{h} v_{h}\right\}_{\omega} \cdot \mathrm{n}_{F} \llbracket w \rrbracket= & \int_{F}\left(\omega_{1} \kappa_{1}^{1 / 2} a_{1}+\omega_{2} \kappa_{2}^{1 / 2} a_{2}\right) \llbracket w \rrbracket \\
\leq & \left(\frac{1}{2} h_{F}\left(\left\|a_{1}\right\|_{L^{2}(F)}^{2}+\left\|a_{2}\right\|_{L^{2}(F)}^{2}\right)\right)^{1 / 2} \\
& \times\left(2\left(\omega_{1}^{2} \kappa_{1}+\omega_{2}^{2} \kappa_{2}\right) h_{F}^{-1}\|\llbracket w \rrbracket\|_{L^{2}(F)}^{2}\right)^{1 / 2}
\end{aligned}
$$

and since $2\left(\omega_{1}^{2} \kappa_{1}+\omega_{2}^{2} \kappa_{2}\right)=\gamma_{\kappa, F}$, it is inferred that

$$
\int_{F}\left\{\kappa \nabla_{h} v_{h}\right\}_{\omega} \cdot \mathrm{n}_{F} \llbracket w \rrbracket \leq\left(\frac{1}{2} h_{F}\left(\left\|a_{1}\right\|_{L^{2}(F)}^{2}+\left\|a_{2}\right\|_{L^{2}(F)}^{2}\right)\right)^{1 / 2}|w|_{\mathrm{J}, \kappa, F} .
$$

Moreover, for all $F \in \mathcal{F}_{h}^{\mathrm{b}}$ with $F=\partial T \cap \partial \Omega$ and $a=\left.\left(\kappa^{1 / 2} \nabla_{h} v_{h}\right)\right|_{T} \cdot \mathrm{n}_{F}$,

$$
\int_{F}\left\{\kappa \nabla_{h} v_{h}\right\}_{\omega} \cdot \mathrm{n}_{F} \llbracket w \rrbracket \leq h_{F}^{1 / 2}\|a\|_{L^{2}(F)}|w|_{\mathrm{J}, \kappa, F} .
$$

Summing over the mesh faces, using the Cauchy-Schwarz inequality, and regrouping the face contributions of each mesh element yields (12).

(ii) Proof of (13). Let $\left(v, w_{h}\right) \in V_{\dagger h} \times V_{h}$. For all $F \in \mathcal{F}_{h}^{\text {i }}$, letting now $a_{i}=$ $\left.\kappa_{i}^{1 / 2}\left(\nabla_{h} v\right)\right|_{T_{i}} \cdot \mathrm{n}_{F}, i \in\{1,2\}$, Hölder's inequality yields

$$
\begin{aligned}
\int_{F}\left\{\kappa \nabla_{h} v\right\}_{\omega} \cdot \mathrm{n}_{F} \llbracket w_{h} \rrbracket \leq & \left(\frac{1}{2} h_{F}^{p \beta_{p}}\left(\left\|a_{1}\right\|_{L^{p}(F)}^{p}+\left\|a_{2}\right\|_{L^{p}(F)}^{p}\right)\right)^{1 / p} \\
& \times 2^{1 / p}\left(\left(\omega_{1}^{q} \kappa_{1}^{q / 2}+\omega_{2}^{q} \kappa_{2}^{q / 2}\right) h_{F}^{-q \beta_{p}}\left\|\llbracket w_{h} \rrbracket\right\|_{L^{q}(F)}^{q}\right)^{1 / q} .
\end{aligned}
$$

We observe that since $q \geq 2$,

$$
\left(\omega_{1}^{q} \kappa_{1}^{q / 2}+\omega_{2}^{q} \kappa_{2}^{q / 2}\right)=\frac{\left(\kappa_{1} \kappa_{2}\right)^{q / 2}}{\left(\kappa_{1}+\kappa_{2}\right)^{q}}\left(\kappa_{1}^{q / 2}+\kappa_{2}^{q / 2}\right) \leq \frac{\left(\kappa_{1} \kappa_{2}\right)^{q / 2}}{\left(\kappa_{1}+\kappa_{2}\right)^{q}}\left(\kappa_{1}+\kappa_{2}\right)^{q / 2}=2^{-q / 2} \gamma_{\kappa, F}^{q / 2} .
$$


Moreover, owing to the inverse inequality (10) and since $\frac{1}{p}+\frac{1}{q}=1$,

$$
h_{F}^{-\beta_{p}}\left\|\llbracket w_{h} \rrbracket\right\|_{L^{q}(F)} \leq C_{q} h_{F}^{-\beta_{p}} h_{F}^{(d-1)\left(\frac{1}{q}-\frac{1}{2}\right)}\left\|\llbracket w_{h} \rrbracket\right\|_{L^{2}(F)}=C_{q} h_{F}^{-1 / 2}\left\|\llbracket w_{h} \rrbracket\right\|_{L^{2}(F)} .
$$

Hence, since $2^{\frac{1}{p}-\frac{1}{2}}=2^{\frac{1}{2}-\frac{1}{q}}$,

$$
\int_{F}\left\{\kappa \nabla_{h} v\right\}_{\omega} \cdot \mathrm{n}_{F} \llbracket w_{h} \rrbracket \leq\left(\frac{1}{2} h_{F}^{p \beta_{p}}\left(\left\|a_{1}\right\|_{L^{p}(F)}^{p}+\left\|a_{2}\right\|_{L^{p}(F)}^{p}\right)\right)^{1 / p} \times 2^{\frac{1}{2}-\frac{1}{q}} C_{q}\left|w_{h}\right|_{\mathrm{J}, \kappa, F} .
$$

Moreover, for all $F \in \mathcal{F}_{h}^{\mathrm{b}}$, proceeding as above with $a=\left.\left(\kappa^{1 / 2} \nabla_{h} v\right)\right|_{T} \cdot \mathrm{n}_{F}$ yields

$$
\int_{F}\left\{\kappa \nabla_{h} v\right\}_{\omega} \cdot \mathrm{n}_{F} \llbracket w_{h} \rrbracket \leq\left(h_{F}^{p \beta_{p}}\|a\|_{L^{p}(F)}^{p}\right)^{1 / p} \times C_{q}\left|w_{h}\right|_{\mathrm{J}, \kappa, F} .
$$

Summing over mesh faces, applying one last time Hölder's inequality, and regrouping the face contributions of each mesh element (since $1 \leq 2^{\frac{1}{2}-\frac{1}{q}}$ for boundary faces), we infer

$$
\left|\sum_{F \in \mathcal{F}_{h}} \int_{F}\left\{\kappa \nabla_{h} v\right\}_{\omega} \cdot \mathrm{n}_{F} \llbracket w_{h} \rrbracket\right| \leq|v|_{\dagger, \kappa, p} \times 2^{\frac{1}{2}-\frac{1}{q}} C_{q}\left(\sum_{F \in \mathcal{F}_{h}}\left|w_{h}\right|_{\mathrm{J}, \kappa, F}^{q}\right)^{1 / q},
$$

and since $q \geq 2$, we obtain

$$
\left(\sum_{F \in \mathcal{F}_{h}}\left|w_{h}\right|_{\mathrm{J}, \kappa, F}^{q}\right)^{1 / q} \leq\left(\sum_{F \in \mathcal{F}_{h}}\left|w_{h}\right|_{\mathrm{J}, \kappa, F}^{2}\right)^{1 / 2}=\left|w_{h}\right|_{\mathrm{J}, \kappa},
$$

thereby yielding (13).

\subsection{Consistency.}

Lemma 3.2 (Jumps of exact solution). The exact solution $u$ is such that

$$
\begin{aligned}
\llbracket u \rrbracket=0 & \forall F \in \mathcal{F}_{h}, \\
\llbracket \kappa \nabla u \rrbracket \cdot \mathrm{n}_{F}=0 & \forall F \in \mathcal{F}_{h}^{\mathrm{i}} .
\end{aligned}
$$

Proof. Property (14) is classical for functions in $H_{0}^{1}(\Omega)$. To prove (15), let $\varphi \in$ $C_{0}^{\infty}(\Omega)$. Since $-\nabla \cdot(\kappa \nabla u)=f \in L^{2}(\Omega)$,

$$
\int_{\Omega}(-\nabla \cdot(\kappa \nabla u)) \varphi=\int_{\Omega} \kappa \nabla u \cdot \nabla \varphi .
$$

Furthermore, we obtain using the Green theorem and (6), for all $T \in \mathcal{T}_{h}$,

$$
\int_{T}(-\nabla \cdot(\kappa \nabla u)) \varphi=\int_{T} \kappa \nabla u \cdot \nabla \varphi-\int_{\partial T}\left(\kappa \nabla u \cdot \mathrm{n}_{T}\right) \varphi .
$$

Summing over mesh elements and accounting for the fact that $\varphi$ vanishes on $\partial \Omega$ yields

$$
\sum_{F \in \mathcal{F}_{h}^{\mathrm{i}}} \int_{F}\left(\llbracket \kappa \nabla u \rrbracket \cdot \mathrm{n}_{F}\right) \varphi=0,
$$

whence the assertion is inferred by choosing the support of $\varphi$ covering a single interface and using a density argument.

Lemma 3.3 (Consistency). For all $w_{h} \in V_{h}$,

$$
a_{h}\left(u, w_{h}\right)=\int_{\Omega} f w_{h} .
$$


Proof. Plug $u$ into the first argument of the bilinear form $a_{h}$ given by (4). Integrating by parts the first term yields

$$
\int_{\Omega} \kappa \nabla u \cdot \nabla_{h} w_{h}=-\sum_{T \in \mathcal{T}_{h}} \int_{T} \nabla \cdot(\kappa \nabla u) w_{h}+\sum_{T \in \mathcal{T}_{h}} \int_{\partial T} \kappa\left(\nabla u \cdot \mathrm{n}_{T}\right) w_{h} .
$$

Rewriting the second term on the right-hand side of the above expression as a sum over mesh faces leads to

$$
\sum_{T \in \mathcal{T}_{h}} \int_{\partial T} \kappa\left(\nabla u \cdot \mathrm{n}_{T}\right) w_{h}=\sum_{F \in \mathcal{F}_{h}^{\text {j }}} \int_{F} \llbracket(\kappa \nabla u) w_{h} \rrbracket \cdot \mathrm{n}_{F}+\sum_{F \in \mathcal{F}_{h}^{\mathrm{b}}} \int_{F} \kappa(\nabla u \cdot \mathrm{n}) w_{h} .
$$

We now observe that for all $F \in \mathcal{F}_{h}^{\text {i }}$,

$$
\llbracket(\kappa \nabla u) w_{h} \rrbracket=\{\kappa \nabla u\}_{\omega} \llbracket w_{h} \rrbracket+\llbracket \kappa \nabla u \rrbracket\left\{w_{h}\right\}_{\bar{\omega}},
$$

where $\left\{w_{h}\right\}_{\bar{\omega}}:=\left.\omega_{T_{2}, F} w_{h}\right|_{T_{1}}+\left.\omega_{T_{1}, F} w_{h}\right|_{T_{2}}$. To prove this identity, we set $a_{i}=$ $\left.(\kappa \nabla u)\right|_{T_{i}}, b_{i}=\left.w_{h}\right|_{T_{i}}, \omega_{i}=\omega_{T_{i}, F}, i \in\{1,2\}$, so that

$$
\begin{aligned}
\llbracket(\kappa \nabla u) w_{h} \rrbracket & =a_{1} b_{1}-a_{2} b_{2} \\
& =\left(\omega_{1} a_{1}+\omega_{2} a_{2}\right)\left(b_{1}-b_{2}\right)+\left(a_{1}-a_{2}\right)\left(\omega_{2} b_{1}+\omega_{1} b_{2}\right) \\
& =\{\kappa \nabla u\}_{\omega} \llbracket w_{h} \rrbracket+\llbracket \kappa \nabla u \rrbracket\left\{w_{h}\right\}_{\bar{\omega}},
\end{aligned}
$$

since $\omega_{1}+\omega_{2}=1$. As a result, accounting for boundary faces,

$$
\sum_{T \in \mathcal{T}_{h}} \int_{\partial T} \kappa\left(\nabla u \cdot \mathrm{n}_{T}\right) w_{h}=\sum_{F \in \mathcal{F}_{h}} \int_{F}\{\kappa \nabla u\}_{\omega} \cdot \mathrm{n}_{F} \llbracket w_{h} \rrbracket+\sum_{F \in \mathcal{F}_{h}^{\mathrm{i}}} \int_{F} \llbracket \kappa \nabla u \rrbracket \cdot \mathrm{n}_{F}\left\{w_{h}\right\}_{\bar{\omega}} .
$$

Combining this expression with (4) and (16) yields

$$
\begin{aligned}
a_{h}\left(u, w_{h}\right)= & -\sum_{T \in \mathcal{T}_{h}} \int_{T} \nabla \cdot(\kappa \nabla u) w_{h}+\sum_{F \in \mathcal{F}_{h}} \eta \frac{\gamma_{\kappa, F}}{h_{F}} \int_{F} \llbracket u \rrbracket \llbracket w_{h} \rrbracket \\
& +\sum_{F \in \mathcal{F}_{h}^{\mathfrak{j}}} \int_{F} \llbracket \kappa \nabla u \rrbracket \cdot \mathrm{n}_{F}\left\{w_{h}\right\}_{\bar{\omega}}-\sum_{F \in \mathcal{F}_{h}} \int_{F} \llbracket u \rrbracket\left\{\kappa \nabla_{h} w_{h}\right\}_{\omega} \cdot \mathrm{n}_{F} .
\end{aligned}
$$

This yields the assertion owing to (14)-(15) and to $-\nabla \cdot(\kappa \nabla u)=f$ in $\Omega$.

3.3. Stability. We now establish the discrete coercivity of the SWIP bilinear form under the usual assumption that the penalty parameter $\eta$ is large enough. An important point is that the minimal threshold on the penalty parameter is independent of the diffusion coefficient.

Lemma 3.4 (Discrete coercivity). For all $\eta>C_{\mathrm{tr}}^{2} N_{\partial}$, the SWIP bilinear form $a_{h}$ is coercive on $V_{h}$ with respect to the $\|\cdot\|_{\kappa}$-norm, i.e.,

$$
\forall v_{h} \in V_{h}, \quad a_{h}\left(v_{h}, v_{h}\right) \geq C_{\text {sta }}\left\|v_{h}\right\|_{\kappa}^{2},
$$

with $C_{\text {sta }}:=\left(\eta-C_{\mathrm{tr}}^{2} N_{\partial}\right)\left\{\max \left(1 / 2, \eta+C_{\mathrm{tr}}^{2} N_{\partial}\right)\right\}^{-1}$.

Proof. Let $v_{h} \in V_{h}$. We first observe that

$$
a_{h}\left(v_{h}, v_{h}\right)=\left\|\kappa^{1 / 2} \nabla_{h} v_{h}\right\|_{\left[L^{2}(\Omega)\right]^{d}}^{2}-2 \sum_{F \in \mathcal{F}_{h}} \int_{F}\left\{\kappa \nabla_{h} v_{h}\right\}_{\omega} \cdot \mathrm{n}_{F} \llbracket v_{h} \rrbracket+\eta\left|v_{h}\right|_{\mathrm{J}, \kappa}^{2},
$$

and bound the second term on the right-hand side using (12) to obtain

$$
a_{h}\left(v_{h}, v_{h}\right) \geq\left\|\kappa^{1 / 2} \nabla_{h} v_{h}\right\|_{\left[L^{2}(\Omega)\right]^{d}}^{2}-2\left|v_{h}\right|_{\dagger, \kappa, 2}\left|v_{h}\right|_{\mathrm{J}, \kappa}+\eta\left|v_{h}\right|_{\mathrm{J}, \kappa}^{2} .
$$


Owing to the discrete trace inequality (11), we readily infer

$$
\left|v_{h}\right|_{\dagger, \kappa, 2} \leq C_{\mathrm{tr}} N_{\partial}^{1 / 2}\left\|\kappa^{1 / 2} \nabla_{h} v_{h}\right\|_{\left[L^{2}(\Omega)\right]^{d}} .
$$

Using the inequality $2 a b \leq \epsilon a^{2}+(1 / \epsilon) b^{2}$ valid for any $\epsilon>0$ yields

$$
a_{h}\left(v_{h}, v_{h}\right) \geq\left(1-C_{\mathrm{tr}}^{2} N_{\partial} \epsilon\right)\left\|\kappa^{1 / 2} \nabla_{h} v_{h}\right\|_{\left[L^{2}(\Omega)\right]^{d}}^{2}+(\eta-1 / \epsilon)\left|v_{h}\right|_{\mathrm{J}, \kappa}^{2} .
$$

It now suffices to take $\epsilon=2\left(\eta+C_{\mathrm{tr}}^{2} N_{\partial}\right)^{-1}$ to infer the assertion.

As a straightforward consequence of the Lax-Milgram Lemma, Lemma 3.4 yields the well-posedness of the discrete problem (5).

3.4. Boundedness. We consider the following additional norm: For all $v \in V_{\uparrow h}$,

$$
\|v\|_{\kappa, \uparrow}:=\|v\|_{\kappa}+|v|_{\dagger, \kappa, p} \text {. }
$$

Lemma 3.5 (Boundedness). There holds

$$
\forall\left(v, w_{h}\right) \in V_{\dagger h} \times V_{h}, \quad a_{h}\left(v, w_{h}\right) \leq C_{\mathrm{bnd}}\|v\|_{\kappa, \uparrow}\left\|w_{h}\right\|_{\kappa} .
$$

with $C_{\mathrm{bnd}}=1+\eta+2^{\frac{1}{2}-\frac{1}{q}} C_{q}+C_{\mathrm{tr}} N_{\partial}^{1 / 2}$

Proof. Let $\left(v, w_{h}\right) \in V_{\uparrow h} \times V_{h}$ and denote by $\mathfrak{T}_{1}, \ldots, \mathfrak{T}_{4}$ the four terms on the right-hand side of (4). Using the Cauchy-Schwarz inequality yields

$$
\left|\mathfrak{T}_{1}+\mathfrak{T}_{2}\right| \leq(1+\eta)\|v\|_{\kappa}\left\|w_{h}\right\|_{\kappa} \leq(1+\eta)\|v\|_{\kappa, \dagger}\left\|w_{h}\right\|_{\kappa} .
$$

Moreover, owing to the bound (13),

$$
\left|\mathfrak{T}_{3}\right| \leq 2^{\frac{1}{2}-\frac{1}{q}} C_{q}|v|_{\dagger, \kappa, p}\left|w_{h}\right|_{\mathrm{J}, \kappa} \leq 2^{\frac{1}{2}-\frac{1}{q}} C_{q}\|v\|_{\kappa, \dagger}\left\|w_{h}\right\|_{\kappa} .
$$

Finally, using the bounds (12) and (17) leads to

$$
\left|\mathfrak{T}_{4}\right| \leq|v|_{\mathrm{J}, \kappa}\left|w_{h}\right|_{\dagger, \kappa, 2} \leq C_{\mathrm{tr}} N_{\partial}^{1 / 2}|v|_{\mathrm{J}, \kappa}\left\|\kappa^{1 / 2} \nabla_{h} w_{h}\right\|_{\left[L^{2}(\Omega)\right]^{d}} \leq C_{\mathrm{tr}} N_{\partial}^{1 / 2}\|v\|_{\kappa}\left\|w_{h}\right\|_{\kappa} .
$$

Collecting the above bounds yields the assertion.

\subsection{Convergence.}

Theorem 3.6 $\left(\|\cdot\|_{\kappa}\right.$-norm error estimate). Assume $\eta>C_{\mathrm{tr}}^{2} N_{\partial}$. There holds

$$
\left\|u-u_{h}\right\|_{\kappa} \leq C \inf _{y_{h} \in V_{h}}\left\|u-y_{h}\right\|_{\kappa, \uparrow},
$$

with $C=1+C_{\mathrm{sta}}^{-1} C_{\mathrm{bnd}}$. Moreover, recalling the definition (7) of $\alpha$,

$$
\left\|u-u_{h}\right\|_{\kappa} \lesssim\left(\sum_{T \in \mathcal{T}_{h}}\|\kappa\|_{L^{\infty}(T)}^{\frac{p}{2}} h_{T}^{p \alpha}\|u\|_{W^{2, p}(T)}^{p}\right)^{1 / p},
$$

yielding, in particular,

$$
\left\|u-u_{h}\right\|_{\kappa} \lesssim \bar{\lambda}^{1 / 2} h^{\alpha}\|u\|_{W^{2, p}\left(\mathcal{T}_{h}\right)} .
$$

Proof. (i) Proof of (18). Let $y_{h} \in V_{h}$. Owing to discrete stability and consistency,

$$
\left\|u_{h}-y_{h}\right\|_{\kappa} \leq C_{\text {sta }}^{-1} \sup _{w_{h} \in V_{h} \backslash\{0\}} \frac{a_{h}\left(u_{h}-y_{h}, w_{h}\right)}{\left\|w_{h}\right\|_{\kappa}}=C_{\text {sta }}^{-1} \sup _{w_{h} \in V_{h} \backslash\{0\}} \frac{a_{h}\left(u-y_{h}, w_{h}\right)}{\left\|w_{h}\right\|_{\kappa}} .
$$

Hence, owing to boundedness,

$$
\left\|u_{h}-y_{h}\right\|_{\kappa} \leq C_{\text {sta }}^{-1} C_{\text {bnd }}\left\|u-y_{h}\right\|_{\kappa, \uparrow} .
$$

Estimate (18) then results from the triangle inequality, the fact that $\left\|u-y_{h}\right\|_{\kappa} \leq$ $\left\|u-y_{h}\right\|_{\kappa, \uparrow}$, and that $y_{h}$ is arbitrary in $V_{h}$. 
(ii) To prove (19), we use (18) with $y_{h}=\pi_{h} u$ where $\pi_{h}$ denotes the $L^{2}$-orthogonal projection onto $V_{h}$. For all $T \in \mathcal{T}_{h}$, using the Sobolev embedding $W^{1, p}(T) \hookrightarrow L^{2}(T)$ since $p>\frac{2 d}{d+2}$ together with interpolation properties in $W^{2, p}(T)$, it can be shown that

$$
h_{T}^{-1}\left\|u-y_{h}\right\|_{L^{2}(T)}+\left\|\nabla_{h}\left(u-y_{h}\right)\right\|_{\left[L^{2}(T)\right]^{d}} \lesssim h_{T}^{1+d\left(\frac{1}{2}-\frac{1}{p}\right)}\|u\|_{W^{2, p}(T)} .
$$

Hence, since $\gamma_{\kappa, F} \leq \min \left(\left.\kappa\right|_{T_{1}},\left.\kappa\right|_{T_{2}}\right)$ for all $F \in \mathcal{F}_{h}^{\mathrm{i}}$ with $F=\partial T_{1} \cap \partial T_{2}, \llbracket y_{h} \rrbracket=$ $\llbracket u-y_{h} \rrbracket$ on all $F \in \mathcal{F}_{h}$, and $\|v\|_{L^{2}(F)} \lesssim\|v\|_{L^{2}(T)}^{1 / 2}\|v\|_{H^{1}(T)}^{1 / 2}$ for all $T \in \mathcal{T}_{h}, F \in \mathcal{F}_{T}$, and $v \in H^{1}(T)$, we infer

$$
\begin{aligned}
\left\|u-y_{h}\right\|_{\kappa} & \lesssim\left(\sum_{T \in \mathcal{T}_{h}}\|\kappa\|_{L^{\infty}(T)} h_{T}^{2+d\left(1-\frac{2}{p}\right)}\|u\|_{W^{2, p}(T)}^{2}\right)^{1 / 2} \\
& \leq\left(\sum_{T \in \mathcal{T}_{h}}\|\kappa\|_{L^{\infty}(T)}^{\frac{p}{2}} h_{T}^{p\left[1+d\left(\frac{1}{2}-\frac{1}{p}\right)\right]}\|u\|_{W^{2, p}(T)}^{p}\right)^{1 / p},
\end{aligned}
$$

since for non-negative real numbers $\left(a_{T}\right)_{T \in \mathcal{T}_{h}},\left(\sum_{T \in \mathcal{T}_{h}} a_{T}^{2}\right)^{1 / 2} \leq\left(\sum_{T \in \mathcal{T}_{h}} a_{T}^{p}\right)^{1 / p}$. Moreover, since $\|v\|_{L^{p}(F)} \lesssim\|v\|_{L^{p}(T)}^{1-\frac{1}{p}}\|v\|_{W^{1, p}(T)}^{\frac{1}{p}}$ for all $T \in \mathcal{T}_{h}, F \in \mathcal{F}_{T}$, and $v \in W^{1, p}(T)$, we infer using interpolation properties in $W^{2, p}(T)$ that

$$
\left\|\left.\nabla\left(u-y_{h}\right)\right|_{T} \cdot \mathrm{n}_{F}\right\|_{L^{p}(F)} \lesssim h_{T}^{1-\frac{1}{p}}\|u\|_{W^{2, p}(T)},
$$

whence, using again $\left(a_{T}\right)_{T \in \mathcal{T}_{h}},\left(\sum_{T \in \mathcal{T}_{h}} a_{T}^{2}\right)^{1 / 2} \leq\left(\sum_{T \in \mathcal{T}_{h}} a_{T}^{p}\right)^{1 / p}$,

$$
\left|u-y_{h}\right|_{\dagger, \kappa, 2} \lesssim\left(\sum_{T \in \mathcal{T}_{h}}\|\kappa\|_{L^{\infty}(T)}^{\frac{p}{2}} h_{T}^{p\left[1+d\left(\frac{1}{2}-\frac{1}{p}\right)\right]}\|u\|_{W^{2, p}(T)}^{p}\right)^{1 / p} .
$$

The proof is complete since $\alpha=1+d\left(\frac{1}{2}-\frac{1}{p}\right)$.

\section{Convergence analysis in 3D FOR $p \in(1,6 / 5]$}

We treat here the $3 \mathrm{~d}$ case with $p \in(1,6 / 5]$. In this case, the regularity (2) is insufficient to establish convergence rates by proceeding as in the previous section. To prove convergence still using admissible mesh sequences (and in particular, shaperegular meshes), we consider here a different analysis technique, inspired by [5] and relying on a compactness argument. In this case, a weaker form of consistency is invoked, which does not require to extend the discrete bilinear form to the continuous space, thereby making the spaces $V_{\dagger}$ and $V_{\dagger h}$ unnecessary.

4.1. Lifting and discrete gradients. An important ingredient of the analysis is a discrete gradient featuring suitable convergence properties for sequences of smooth and of discrete functions. The discrete gradient is defined in terms of the weighted lifting operators introduced by Di Pietro, Ern, and Guermond [6]; see also Agélas, Di Pietro, Eymard, and Masson [1]. More precisely, for any integer $l \geq 0$ and all $F \in \mathcal{F}_{h}$, we define the linear operator $\mathrm{r}_{\omega, F}^{l}: L^{2}(F) \rightarrow\left[\mathbb{P}_{d}^{l}\left(\mathcal{T}_{h}\right)\right]^{d}$ such that, for all $v \in L^{2}(F)$,

$$
\int_{\Omega} \mathrm{r}_{\omega, F}^{l}(v) \cdot \tau_{h}=\int_{F} v\left\{\tau_{h}\right\}_{\omega} \cdot \mathrm{n}_{F} \quad \forall \tau_{h} \in\left[\mathbb{P}_{d}^{l}\left(\mathcal{T}_{h}\right)\right]^{d}
$$


We also define the following global lifting:

$$
\mathrm{R}_{\omega, h}^{l}(v):=\sum_{F \in \mathcal{F}_{h}} \mathrm{r}_{\omega, F}^{l}(v)
$$

The $L^{2}$-norm of the global lifting can be bounded in terms of the jump seminorm. Proceeding as in the proof of (12) yields that for all $l \geq 0$ and all $v \in V_{\dagger}$,

$$
\left\|\kappa^{1 / 2} \mathrm{R}_{\omega, h}^{l}(\llbracket v \rrbracket)\right\|_{\left[L^{2}(\Omega)\right]^{d}} \leq C_{\mathrm{tr}} N_{\partial}^{1 / 2}|v|_{\mathrm{J}, \kappa} .
$$

For all $l \geq 0$ and all $v_{h} \in V_{h}$, we define the discrete gradient

$$
\mathrm{G}_{\omega, h}^{l}\left(v_{h}\right):=\nabla_{h} v_{h}-\mathrm{R}_{\omega, h}^{l}\left(\llbracket v_{h} \rrbracket\right) .
$$

For future use, we also introduce the following data-independent norms,

$$
\|v\|:=\left(\left\|\nabla_{h} v\right\|_{\left[L^{2}(\Omega)\right]^{d}}^{2}+|v|_{J}^{2}\right)^{1 / 2}, \quad|v|_{J}:=\left(\sum_{F \in \mathcal{F}_{h}} h_{F}^{-1}\|\llbracket v \rrbracket\|_{L^{2}(F)}^{2}\right)^{1 / 2} .
$$

For every integer $l \geq 0$, we also denote by $\pi_{h}^{l}$ the $L^{2}$-orthogonal projection onto $\mathbb{P}_{d}^{l}\left(\mathcal{T}_{h}\right)$; the same notation is used for the $L^{2}$-orthogonal componentwise projection onto $\left[\mathbb{P}_{d}^{l}\left(\mathcal{T}_{h}\right)\right]^{d}$.

Lemma 4.1 (Discrete Rellich-Kondrachov). Let $\left(v_{h}\right)_{h \in \mathcal{H}}$ be a sequence in $\left(V_{h}\right)_{h \in \mathcal{H}}$, uniformly bounded in the $\|\cdot\|-n o r m$. Then, there exists a function $v \in H_{0}^{1}(\Omega)$ such that as $h \rightarrow 0$, up to a subsequence, $v_{h} \rightarrow v$ strongly in $L^{2}(\Omega)$.

Proof. See [5, Theorem 6.3].

Lemma 4.2 (Properties of $\mathrm{G}_{\omega, h}^{l}$ ). The discrete gradients $\mathrm{G}_{\omega, h}^{l}, l \geq 0$, enjoy the following properties:

(i) For all sequences $\left(v_{h}\right)_{h \in \mathcal{H}}$ in $\left(V_{h}\right)_{h \in \mathcal{H}}$ uniformly bounded in the $\|\cdot\|-n o r m$, as $h \rightarrow 0, \mathrm{G}_{\omega, h}^{l}\left(v_{h}\right) \rightarrow \nabla v$ weakly in $\left[L^{2}(\Omega)\right]^{d}$ with $v \in H_{0}^{1}(\Omega)$ provided by Theorem 4.1;

(ii) For all $\varphi \in C_{0}^{\infty}(\Omega)$, as $h \rightarrow 0, \mathrm{G}_{\omega, h}^{l}\left(\pi_{h}^{1} \varphi\right) \rightarrow \nabla \varphi$ strongly in $\left[L^{2}(\Omega)\right]^{d}$.

Proof. (i) To prove the weak convergence of $\mathrm{G}_{\omega, h}^{l}\left(v_{h}\right)$ to $\nabla v$, let $\Phi \in\left[C_{0}^{\infty}(\Omega)\right]^{d}$, set $\Phi_{h}:=\pi_{h}^{l} \Phi$ and observe that

$$
\begin{aligned}
\int_{\Omega} \mathrm{G}_{\omega, h}^{l}\left(v_{h}\right) \cdot \Phi & =-\int_{\Omega} v_{h} \nabla \cdot \Phi+\sum_{T \in \mathcal{T}_{h}} \int_{\partial T} v_{h} \Phi \cdot \mathrm{n}_{T}-\sum_{F \in \mathcal{F}_{h}} \int_{\Omega} \mathrm{r}_{\omega, F}^{l}\left(\llbracket v_{h} \rrbracket\right) \cdot \Phi_{h} \\
& =-\int_{\Omega} v_{h} \nabla \cdot \Phi+\sum_{F \in \mathcal{F}_{h}} \int_{F} \llbracket v_{h} \rrbracket\left\{\Phi-\Phi_{h}\right\}_{\omega} \cdot \mathrm{n}_{F}=\mathfrak{T}_{1}+\mathfrak{T}_{2},
\end{aligned}
$$

where we have used the definition of the $L^{2}$-orthogonal projection together with (20) and (21). As $h \rightarrow 0, \mathfrak{T}_{1} \rightarrow-\int_{\Omega} v \nabla \cdot \Phi$. For the second term, the Cauchy-Schwarz inequality yields

$$
\mathfrak{T}_{2} \leq\left|v_{h}\right|_{\mathrm{J}, \kappa} \times\left(\sum_{F \in \mathcal{F}_{h}} h_{F} \gamma_{F}^{-1} \int_{F}\left|\left\{\Phi-\Phi_{h}\right\}_{\omega}\right|^{2}\right)^{1 / 2},
$$

which tends to zero owing to the approximation properties of the $L^{2}$-orthogonal projection together with the fact that $\left|v_{h}\right|_{\mathrm{J}, \kappa} \leq \bar{\lambda}^{1 / 2}\left|v_{h}\right|_{\mathrm{J}}$ is uniformly bounded by assumption. This concludes the proof. 
(ii) Let $\varphi_{h}:=\pi_{h}^{1} \varphi$. Then, $\mathrm{G}_{\omega, h}^{l}\left(\varphi_{h}\right)=\nabla_{h} \varphi_{h}-\mathrm{R}_{\omega, h}^{l}\left(\llbracket \varphi_{h} \rrbracket\right)=\mathfrak{T}_{1}+\mathfrak{T}_{2}$. Clearly, $\mathfrak{T}_{1} \rightarrow \nabla \varphi$ strongly in $\left[L^{2}(\Omega)\right]^{d}$ as $h \rightarrow 0$. Moreover, owing to (22), it is inferred that $\left\|\mathrm{R}_{\omega, h}^{l}\left(\llbracket \varphi_{h} \rrbracket\right)\right\|_{\left[L^{2}(\Omega)\right]^{d}} \leq C_{\mathrm{tr}} N_{\partial}^{1 / 2}\left|\varphi_{h}\right|_{\mathrm{J}, \kappa}=C_{\mathrm{tr}} N_{\partial}^{1 / 2}\left|\varphi_{h}-\varphi\right|_{\mathrm{J}, \kappa}$, which tends to zero as $h \rightarrow 0$, thereby concluding the proof.

4.2. Convergence. The SWIP bilinear form $a_{h}$ admits the following equivalent formulation on $V_{h} \times V_{h}$ : For $l \in\{k-1, k\}$,

$$
a_{h}\left(v_{h}, w_{h}\right)=\int_{\Omega} \kappa \mathrm{G}_{\omega, h}^{l}\left(v_{h}\right) \cdot \mathrm{G}_{\omega, h}^{l}\left(w_{h}\right)+j_{h}\left(v_{h}, w_{h}\right),
$$

with $j_{h}\left(v_{h}, w_{h}\right):=-\int_{\Omega} \kappa \mathrm{R}_{\omega, h}^{l}\left(\llbracket v_{h} \rrbracket\right) \cdot \mathrm{R}_{\omega, h}^{l}\left(\llbracket w_{h} \rrbracket\right)+\sum_{F \in \mathcal{F}_{h}} \eta \gamma_{\kappa, F} h_{F}^{-1} \int_{F} \llbracket v_{h} \rrbracket \llbracket w_{h} \rrbracket$. We can now state and prove the main result of this section.

Theorem 4.3 (Convergence to minimal regularity solutions). Let $\left(u_{h}\right)_{h \in \mathcal{H}}$ be the sequence of approximate solutions generated by solving the discrete problems (5). Then, as $h \rightarrow 0$, (i) $u_{h} \rightarrow u$ strongly in $L^{2}(\Omega)$, (ii) $\nabla_{h} u_{h} \rightarrow \nabla u$ strongly in $\left[L^{2}(\Omega)\right]^{d}$, (iii) $\left|u_{h}\right|_{\mathrm{J}} \rightarrow 0$, with $u \in V$ unique solution to (1).

Proof. (i) A priori estimate. We recall the discrete Poincaré inequality [5, eq. (75)],

$$
\forall v_{h} \in V_{h}, \quad\left\|v_{h}\right\|_{L^{2}(\Omega)} \leq \sigma_{2}\left\|v_{h}\right\|,
$$

with $\sigma_{2}$ independent of the mesh-size $h$. Owing to the coercivity of $a_{h}$ together with (25), it is inferred that

$$
\begin{aligned}
C_{\text {sta }} \underline{\lambda}\left\|u_{h}\right\|^{2} \leq C_{\text {sta }}\left\|u_{h}\right\|_{\kappa}^{2} \leq a\left(u_{h}, u_{h}\right) & =\int_{\Omega} f u_{h} \\
& \leq\|f\|_{L^{2}(\Omega)}\left\|u_{h}\right\|_{L^{2}(\Omega)} \leq \sigma_{2}\|f\|_{L^{2}(\Omega)}\left\|u_{h}\right\|,
\end{aligned}
$$

hence $\left\|u_{h}\right\| \leq \sigma_{2}\left(C_{\text {sta }} \underline{\lambda}\right)^{-1}\|f\|_{L^{2}(\Omega)}$, that is to say, the sequence of discrete solutions is uniformly bounded in the $\|\cdot\| \cdot \|$-norm.

(ii) Compactness. Owing to Theorem 4.1 together with Lemma 4.2i, there exists $\bar{u} \in H_{0}^{1}(\Omega)$ such that, as $h \rightarrow 0$, up to a subsequence, $u_{h} \rightarrow \bar{u}$ strongly in $L^{2}(\Omega)$ and $\mathrm{G}_{\omega, h}^{l}\left(u_{h}\right) \rightarrow \nabla \bar{u}$ weakly in $\left[L^{2}(\Omega)\right]^{d}$.

(iii) Identification of the limit. Let $\varphi \in C_{0}^{\infty}(\Omega)$ and set $\varphi_{h}:=\pi_{h}^{1} \varphi$. Owing to the regularity of $\varphi$, it is clear that $\left\|\varphi-\varphi_{h}\right\|_{\kappa} \rightarrow 0$ as $h \rightarrow 0$. Observe that

$$
a_{h}\left(u_{h}, \varphi_{h}\right)=\int_{\Omega} \kappa \mathrm{G}_{\omega, h}^{l}\left(u_{h}\right) \cdot \mathrm{G}_{\omega, h}^{l}\left(\varphi_{h}\right)+j_{h}\left(u_{h}, \varphi_{h}\right)=\mathfrak{T}_{1}+\mathfrak{T}_{2} .
$$

As $h \rightarrow 0, \mathfrak{T}_{1} \rightarrow \int_{\Omega} \kappa \nabla \bar{u} \cdot \nabla \varphi$ owing to the weak convergence of $\mathrm{G}_{\omega, h}^{l}\left(u_{h}\right)$ to $\nabla \bar{u}$ and to the strong convergence of $\mathrm{G}_{\omega, h}^{l}\left(\varphi_{h}\right)$ to $\nabla \varphi$ proved in Lemma 4.2. Furthermore, the Cauchy-Schwarz inequality together with (22) yield

$$
\left|\mathfrak{T}_{2}\right|=\left|j_{h}\left(u_{h}, \varphi_{h}\right)\right| \leq\left(C_{\mathrm{tr}}^{2} N_{\partial}+\eta\right)\left|u_{h}\right|_{\mathrm{J}, \kappa}\left|\varphi_{h}\right|_{\mathrm{J}, \kappa} \leq\left(C_{\mathrm{tr}}^{2} N_{\partial}+\eta\right) \bar{\lambda}^{1 / 2}\left|u_{h}\right|_{\mathrm{J}}\left|\varphi_{h}\right|_{\mathrm{J}, \kappa}
$$

Since $\left|u_{h}\right|_{\mathrm{J}}$ is bounded by point (i), and since $\left|\varphi_{h}\right|_{\mathrm{J}, \kappa}=\left|\varphi_{h}-\varphi\right|_{\mathrm{J}, \kappa}$ tends to zero as $h \rightarrow 0$, it is inferred that $\mathfrak{T}_{2} \rightarrow 0$. As a result,

$$
\int_{\Omega} \kappa \nabla \bar{u} \cdot \nabla \varphi \leftarrow a_{h}\left(u_{h}, \varphi_{h}\right)=\int_{\Omega} f \varphi_{h} \rightarrow \int_{\Omega} f \varphi .
$$

Hence, by the density of $C_{0}^{\infty}(\Omega)$ in $H_{0}^{1}(\Omega), \bar{u}=u$, the unique solution to (1). Owing to the uniqueness of $u$, the whole sequence of discrete solutions converges. 
(iv) Strong convergence of the gradient and of the jumps. Eqs. (22) and (24) yield

$$
\forall v_{h} \in V_{h}, \quad a_{h}\left(v_{h}, v_{h}\right) \geq\left\|\kappa^{1 / 2} \mathrm{G}_{\omega, h}^{l}\left(v_{h}\right)\right\|_{\left[L^{2}(\Omega)\right]^{d}}^{2}+\left(\eta-C_{\mathrm{tr}}^{2} N_{\partial}\right)\left|v_{h}\right|_{\mathrm{J}, \kappa}^{2} .
$$

From the weak convergence of $\mathrm{G}_{\omega, h}^{l}\left(u_{h}\right)$ to $\nabla u$, we readily infer the weak convergence of $\kappa^{1 / 2} \mathrm{G}_{\omega, h}^{l}\left(u_{h}\right)$ to $\kappa^{1 / 2} \nabla u$. Then, owing to (27) and to weak convergence,

$$
\liminf _{h \rightarrow 0} a_{h}\left(u_{h}, u_{h}\right) \geq \liminf _{h \rightarrow 0}\left\|\kappa^{1 / 2} \mathrm{G}_{\omega, h}^{l}\left(u_{h}\right)\right\|_{\left[L^{2}(\Omega)\right]^{d}}^{2} \geq\left\|\kappa^{1 / 2} \nabla u\right\|_{\left[L^{2}(\Omega)\right]^{d}}^{2} .
$$

Furthermore, still owing to (27),

$$
\begin{aligned}
\limsup _{h \rightarrow 0}\left\|\kappa^{1 / 2} \mathrm{G}_{\omega, h}^{l}\left(u_{h}\right)\right\|_{\left[L^{2}(\Omega)\right]^{d}}^{2} & \leq \limsup _{h \rightarrow 0} a_{h}\left(u_{h}, u_{h}\right) \\
& =\limsup _{h \rightarrow 0} \int_{\Omega} f u_{h}=\int_{\Omega} f u=\left\|\kappa^{1 / 2} \nabla u\right\|_{\left[L^{2}(\Omega)\right]^{d}}^{2} .
\end{aligned}
$$

This classically proves the strong convergence of $\kappa^{1 / 2} \mathrm{G}_{\omega, h}^{l}\left(u_{h}\right)$ to $\kappa^{1 / 2} \nabla u$ in $\left[L^{2}(\Omega)\right]^{d}$ and, hence, the strong convergence of $\mathrm{G}_{\omega, h}^{l}\left(u_{h}\right)$ to $\nabla u$ in $\left[L^{2}(\Omega)\right]^{d}$. Note that $a_{h}\left(u_{h}, u_{h}\right) \rightarrow\left\|\kappa^{1 / 2} \nabla u\right\|_{\left[L^{2}(\Omega)\right]^{d}}^{2}$ also. Using (27) we then infer

$$
\left(\eta-C_{\mathrm{tr}}^{2} N_{\partial}\right)\left|u_{h}\right|_{\mathrm{J}, \kappa}^{2} \leq a_{h}\left(u_{h}, u_{h}\right)-\left\|\kappa^{1 / 2} \mathrm{G}_{\omega, h}^{l}\left(u_{h}\right)\right\|_{\left[L^{2}(\Omega)\right]^{d}}^{2},
$$

and, since $\eta>C_{\mathrm{tr}}^{2} N_{\partial}$ and the right-hand side tends to zero, $\left|u_{h}\right|_{\mathrm{J}, \kappa} \rightarrow 0$. To infer that $\left|u_{h}\right|_{\mathrm{J}} \rightarrow 0$, simply observe that $\left|u_{h}\right|_{\mathrm{J}} \leq \underline{\lambda}^{-1 / 2}\left|u_{h}\right|_{\mathrm{J}, \kappa}$.

Remark 4.4. When extended to $V_{\dagger h} \times V_{h}$, the discrete bilinear form $a_{h}$ defined by (24) is no longer consistent in the usual finite element sense; see [5, Remark 3.3]. However, (26) shows that $a_{h}$ retains a form of weak asymptotic consistency which suffices to infer the convergence of the method when $u$ only exhibits the minimal regularity.

Acknowledgment. The authors are grateful to S. Nicaise (Université de Valenciennes) for stimulating discussions. This work was partially supported by the GNR MoMaS (PACEN/CNRS, ANDRA, BRGM, CEA, EdF, IRSN, France).

\section{REFERENCES}

[1] L. Agélas, D. A. Di Pietro, R. Eymard, and R. Masson. An abstract analysis framework for nonconforming approximations of diffusion problems on general meshes. IJFV, 7(1):1-29, 2010.

[2] D. N. Arnold. An interior penalty finite element method with discontinuous elements. SIAM J. Numer. Anal., 19:742-760, 1982.

[3] S. C. Brenner and L. R. Scott. The mathematical theory of finite element methods, volume 15 of Texts in Applied Mathematics. Springer, New York, third edition, 2008.

[4] E. Burman and P. Zunino. A domain decomposition method for partial differential equations with non-negative form based on interior penalties. SIAM J. Numer. Anal., 44:1612-1638, 2006.

[5] D. A. Di Pietro and A. Ern. Discrete functional analysis tools for discontinuous Galerkin methods with application to the incompressible Navier-Stokes equations. Math. Comp., 79:13031330, 2010. DOI: 10.1090/S0025-5718-10-02333-1.

[6] D. A. Di Pietro, A. Ern, and J.-L. Guermond. Discontinuous Galerkin methods for anisotropic semi-definite diffusion with advection. SIAM J. Numer. Anal., 46(2):805-831, 2008.

[7] A. Ern and J.-L. Guermond. Theory and Practice of Finite Elements, volume 159 of Applied Mathematical Sciences. Springer-Verlag, New York, NY, 2004.

[8] A. Ern and A. F. Stephansen. A posteriori energy-norm error estimates for advection-diffusion equations approximated by weighted interior penalty methods. J. Comp. Math., 26(4):488$510,2008$. 
[9] A. Ern, A. F. Stephansen, and P. Zunino. A discontinuous Galerkin method with weighted averages for advection-diffusion equations with locally small and anisotropic diffusivity. IMA J. Numer. Anal., 29(2):235-256, 2009.

[10] B. Heinrich and S. Nicaise. The Nitsche mortar finite-element method for transmission problems with singularities. IMA J. Numer. Anal., 23(2):331-358, 2003.

[11] B. Heinrich and K. Pietsch. Nitsche type mortaring for some elliptic problem with corner singularities. Computing, 68(3):217-238, 2002.

[12] B. Heinrich and K. Pönitz. Nitsche type mortaring for singularly perturbed reaction-diffusion problems. Computing, 75(4):257-279, 2005.

[13] S. Nicaise and A.-M. Sändig. General interface problems. I, II. Math. Methods Appl. Sci., 17(6):395-429, 431-450, 1994.

[14] R. Stenberg. Mortaring by a method of J.A. Nitsche. In Idelsohn S.R., Oñate E., and Dvorkin E.N., editors, Computational Mechanics: New trends and applications, pages 1-6, Barcelona, Spain, 1998. Centro Internacional de Métodos Numéricos en Ingeniería.

[15] A. F. Stephansen. Méthodes de Galerkine discontinues et analyse d'erreur a posteriori pour les problèmes de diffusion hétérogène. Ph.D. thesis, Ecole Nationale des Ponts et Chaussées, 2007.

[16] R. Verfürth. personal communication. 2009.

[17] T. P. Wihler, P. Frauenfelder, and C. Schwab. Exponential convergence of the $h p$-DGFEM for diffusion problems. Comput. Math. Appl., 46:183-205, 2003.

[18] T. P. Wihler and B. Rivière. Discontinuous Galerkin methods for second-order elliptic PDE with low-regularity solutions. J. Sci. Comp., 2010. Published online. DOI: $10.1007 /$ s10915-010-9387-9.

${ }^{2}$ IfP Energies nouvelles, 1 \& 4 avenue de bois Préau, 92852 Rueil-Malmaison Cedex, France

E-mail address: dipietrd@if penergiesnouvelles.fr

${ }^{1}$ Université Paris-Est, Cermics, Ecole des Ponts, 77455 Marne la Vallée Cedex 2, France

E-mail address: ern@cermics.enpc.fr 